\title{
Two types of single-beam deflection and asymmetric transmission in photonic structures without interface corrugations
}

\author{
Andriy E. Serebryannikov, ${ }^{1, *}$ Evrim Colak, ${ }^{2}$ Thore Magath, ${ }^{3}$ and Ekmel Ozbay ${ }^{4}$ \\ ${ }^{1}$ Adam Mickiewicz University, Faculty of Physics, 61-614 Poznan, Poland \\ ${ }^{2}$ Ankara University, Electrical Engineering Department, Golbasi, 06830 Ankara, Turkey \\ ${ }^{3}$ Panasonic Electron Devices Europe GmbH, European Technology Center, D-21337 Luneburg, Germany \\ ${ }^{4}$ Bilkent University, Nanotechnology Research Center-NANOTAM, 06800 Ankara, Turkey \\ ${ }^{*}$ Corresponding author: andser@amu.edu.pl
}

Received 15 August 2016; revised 14 October 2016; accepted 31 October 2016; posted 1 November 2016 (Doc. ID 273334); published 28 November 2016

\begin{abstract}
We study single-beam deflection and asymmetry in transmission, two aspects of the same phenomenon that appear in the topologically simple, nonsymmetric, photonic crystal (PhC)-based structures without corrugations at the interfaces. Strong diffractions enabling efficient blazing, i.e., redistribution of the incident wave energy in favor of the desired higher diffraction order(s), can be achieved owing to the defect-like layer(s) embedded in a regular slab of PhC. The main features, together with the peculiarities of the two basic transmission types and relevant coupling and deflection scenarios, are discussed, for one of which a part of the PhC works in the evanescent-wave regime. Performances are suggested, in which efficient single-beam deflection and asymmetry in transmission can be obtained even when the irregular layer is deeply embedded. More than $97 \%$ of the incident wave energy can be converted into a single deflected beam that is associated with the first negative diffraction order, even though the entire structure is nonsymmetric and the diffractive element is located at some distance from the incidence interface. (c) 2016 Optical Society of America
\end{abstract}

OCIS codes: (050.1940) Diffraction; (050.2770) Gratings; (050.5298) Photonic crystals; (120.7000) Transmission.

https://doi.org/10.1364/JOSAA.33.002450

\section{INTRODUCTION}

Advanced functionality and operation regimes can be realized in photonic structures made of linear, isotropic, passive materials, in which the effects of different periodicities are properly combined. Among them, one should mention those known as photonic crystal $(\mathrm{PhC})$ gratings [1-5]. Initially, $\mathrm{PhC}$ gratings were proposed for the reflection mode $[1,6]$ and later for the transmission mode $[3,7]$. The interest in PhC gratings has been growing in the connection with diffraction-inspired asymmetric transmission [2-5] and locations of frequency-domain thresholds for higher diffraction orders [7], which are unusual compared to the classical grating theory [8]. To obtain these regimes, the spatial inversion symmetry must be broken. The structures for asymmetric transmission are Lorentz reciprocal and, thus, provide regimes of directional selectivity that are distinguished from those achievable in the nonreciprocal structures with the aid of anisotropic materials (see $[9,10])$. Different regimes of diffraction-inspired asymmetric transmission have also been demonstrated in nonsymmetric gratings made of ultralow-index natural materials [11,12], nonsymmetric metamaterial gratings $[13,14]$, and finite-thickness metallic slabs with branched slots creating different periods at two interfaces [15].

The role and scenarios of suppression of the zero order for wideband asymmetric transmission have been studied $[2,4]$. Indeed, for any grating with broken structural symmetry, forward (further indicated by $\rightarrow$ ) and backward (indicated by $\leftarrow)$ transmission is exactly the same for the zero order, i.e., $t_{0}=t_{0}=t_{0}^{\leftarrow}$ due to the reciprocity, but it is different (in the general case) for higher orders, i.e., $t_{m} \neq t_{m}^{\leftarrow}$ when $|m|>0$. Thanks to the latter, we have $T^{\rightarrow} \neq T^{\leftarrow}$, where $T^{\rightarrow}$ and $T^{\leftarrow}$ mean the forward and backward transmittances that include the contributions of all propagating orders. If dispersion block coupling of the zero order to a FloquetBloch wave of $\mathrm{PhC}$ and different corrugations are used at the front-side and the back-side interfaces so coupling is possible only at one of the interfaces, the most interesting regime of asymmetric transmission (known as unidirectional transmission) can be realized due to the common effects of diffraction and dispersion. For this regime, transmission is nonzero only for 
one of the two opposite incidence directions. Additionally, asymmetry in transmission can be achieved owing to the orientation of the interfaces, such as in $\mathrm{PhC}$ prisms $[16,17]$, or the use of two orthogonal polarizations, as is done for coupled metasurfaces built of subwavelength resonators $[18,19]$. Further examples of asymmetric effects include those related to transmission through a subwavelength slit that can be enhanced by the effect of surface waves [20,21], propagation in a waveguide in a PhC slab $[22,23]$, and transmission through graded-index (chirped) PhCs [24]. It can also be obtained by using structures with parity-time symmetry $[25,26]$. Hence, asymmetry in transmission is a very general phenomenon that may enable various operation regimes.

Single-beam unidirectional deflection [4], a regime of asymmetric transmission inspired by diffractions, has been a focus of interest also because of its connection with the classical problem of blazing [27], i.e., the redistribution of the incident wave energy in favor of one of higher diffraction orders. While echelette reflection mode gratings are known as the classical structures that enable blazing, alternative structures have later been proposed, e.g., those based on binary gratings with several ridges and grooves per period and complex gratings with a period containing multiple subwavelength holes and pillars [28-30]. Today, blazing and deflection remain important for the further development of advanced photonic and microwave components and devices. In particular, Huygens metasurfaces have recently been suggested that allow one to obtain single-beam deflection in the reflection-free regime [31,32]. Similar regimes are highly demanded for PhC-based platforms, which are widely used in photonic circuitry. Moreover, the richness of the dispersion types achievable in one $\mathrm{PhC}$ provides the principal possibility of multifunctionality, i.e., different functions can be realized within different frequency ranges in one structure. Until now, single-beam deflection has been studied in PhC-based structures with a corrugated interface that imposes significant restrictions on the resulting design. At the same time, the recent results on dual-beam unidirectional splitting, another regime inspired by diffractions, indicate the possibility of strong diffractions in photonic structures without a corrugated interface [33].

In this paper, our hypothesis about the possibility of strong single-beam deflection and blazing and related asymmetric transmission in topologically simple PhC-based structures without interface corrugations, which may involve evanescent-wave components, is validated and the generality of the basic effects is demonstrated. The required diffractions are obtained in the proposed approach due to the irregular layer of the rods that has a different period than the layers belonging to the regular part of $\mathrm{PhC}$, into which the irregular layer is embedded. Its location is chosen so the entire structure is nonsymmetric, i.e., the necessary condition of asymmetric transmission is fulfilled. The results are presented for well-selected structures that provide illustrative results at different locations of the irregular layer with respect to the interfaces. The main attention will be paid to the case when a sole irregular layer is embedded in a regular PhC. Such defect-type irregular layers are expected to enable deflection in one frequency range and to be utilized for the realization of another functionality, e.g., defect-mode wave guiding [34], in another frequency range but in the same structure. Most of the presented results are related to the transmittance; a brief comparison with the dispersion results is also included. Transmission results are obtained with the aid of the coupled integral equation technique [35], while CST Microwave Studio [36], a full-wave software based on the finite integration method, and a self-made post-processing code were used to compute the dispersion.

\section{GEOMETRY AND OPERATION PRINCIPLES}

Figure 1 presents the general geometry and schematics that illustrate two ideal scenarios of deflection and asymmetric transmission. The studied structures are based on a twodimensional square-lattice PhC [see Fig. 1(a)]. The lattice constant and diameter and permittivity of the rods are denoted by $a, d$, and $\varepsilon$, respectively. The interfaces are assumed to be along the $\Gamma-\mathrm{X}$ direction. The defect-type irregular layer consists of the same rods; it is assumed to be periodic over $x$, with period $p=L=2 a$. This layer is the only diffractive element, while the regular parts of the PhC cannot themselves create diffractions in the considered parameter range. The total number of the rod layers, $N$, is assumed to be even, i.e., the resulting structure with one irregular layer is always nonsymmetric, as required for asymmetric transmission. Strictly speaking, any structure periodic over $x$ (also one made of a homogeneous dielectric) that is nonsymmetric with respect to its midplane in the $y$ direction and enables propagation of higher diffraction orders can show asymmetry in transmission because $t_{m} \neq t_{m}^{\leftarrow} \neq$ 0 at $|m|>0$. As a result, different portions of the incident wave energy are transmitted from one half-space to the other when the difference between two illumination directions is $180^{\circ}$. The asymmetry can be enhanced, for instance, by embedding an additional grating into an initial grating that is made of a homogeneous material and has corrugations only on one side [37]. However, the strongest enhancement can be achieved due to the common effect of diffraction and dispersion, as occurs in $\mathrm{PhC}$ gratings [2-4].

The structure is illuminated by a plane electromagnetic wave that is s-polarized, i.e., the electric field is along the rod axes, and incident at the angle $\theta$. Figures 1 (b) and 1 (c) represent the schematics of two scenarios of ideal single-beam deflection. Figure 1(b) illustrates the scenario in which transmission through a properly designed nonsymmetric structure is possible only for one of two opposite incidence directions, whereas

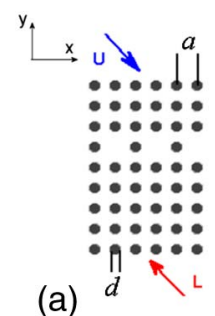

(a)

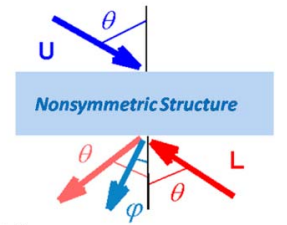

(b)

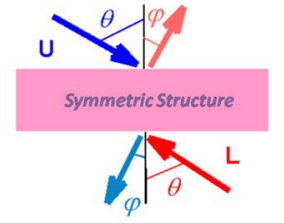

(c)
Fig. 1. (a) General geometry of the PhC-based nonsymmetric structure (three periods over $x$ are shown) and schematics showing scenarios of ideal single-beam deflection in (b) nonsymmetric structure with simultaneous transmission and reflection processes, and (c) symmetric structure with two simultaneous transmission processes. $\mathrm{U}$ and $\mathrm{L}$ stand for upper-side and lower-side illumination, respectively. 
specular reflection occurs for the second direction. In [4], this scenario has been discussed for $\mathrm{PhC}$ gratings with nondeep one-side interface corrugations. However, it is not clear yet how a similar scenario can be realized when the diffractive element is located inside the structure.

According to [4], the main role of dispersion is to block coupling and transmission for an undesired range of the tangential wavenumber and provide coupling for a desired one. In line with Lorentz reciprocity, it can even be realized only due to the effect of diffractions. In contrast with the previous case, it can be done only in a narrow band because of the absence of the wideband blocking effect of dispersion (see [4]).

An important feature illustrated in Fig. 1(b) is that the directions of the outgoing beams for upper-side and lowerside incidence do not coincide in the general case, i.e., $\varphi \neq \theta$, where

$$
\varphi=\phi_{-1}=\arcsin [\sin \theta-2 \pi /(k L)]
$$

is the diffraction angle for the order $m=-1[8] ; k=\omega / c$ is free-space wave number. Thus, two simultaneous processes can be independent, at least if the deflection is ideal. The only exception is related to the case when $\theta=\varphi$, i.e., $\sin \theta=\pi /(k L)$. Then, two beams are combined into one. For comparison, in Fig. 1(c), the ideal blazing is schematically shown for a symmetric structure, in which it may occur for both opposite incidence directions. Even in this case, two processes can be independent, provided the deflection is ideal. This regime is similar to one of those studied in [20] for metallic gratings with nonsymmetric corrugations and a subwavelength slit. From the above given reasons, the advantages of ideal deflection are obvious. In this paper, consideration is restricted to the scenario in Fig. 1(b) and similar but nonideal deflection scenarios.

At least two different types of transmission and deflection are expected to be possible. For the first of them, dispersion of a Floquet-Bloch mode of the $\mathrm{PhC}$ enables coupling of the zero order $(m=0)$ at the incidence interface, so the role of the irregular layer, like that shown in Fig. 1(a), is to redistribute the in-coupled energy in favor of one of the higher orders $(|m|>0)$. In this case, a propagating-wave regime takes place for both upper and lower regular segments of the $\mathrm{PhC}$. For the second one, dispersion does not permit coupling at the incidence interface and, therefore, there is no wave propagating in the regular segment adjacent to the incidence interface. However, it may appear in the regular segment adjacent to the exit interface due to the order(s) $|m|>0$ created by a properly designed irregular layer. These two types are schematically illustrated in Fig. 2. In cases when the irregular layer is not embedded deeply, a part of the entire structure that comprises the regular segment adjacent to the incidence interface and the irregular layer should be considered rather as a complex grating. It is noteworthy that the translation properties of the entire structure result in $t_{m}=t_{-m}$ and $\phi_{m}=-\phi_{-m}$ when $\theta=0$ [8]. Hence, tilting is necessary for single-beam deflection with $t_{-1}>0$, whereas $t_{+1}=0$ and $t_{0}=0$. All further considerations are restricted to structures with $N=12$.

Numerical simulations were carried out to validate the above-described hypothesis regarding single-beam deflection inspired by diffractions that do not require interface corrugations, while evanescent-wave components are either present or
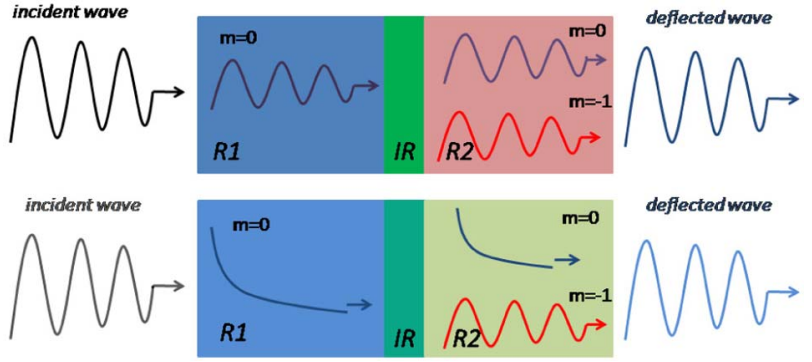

Fig. 2. Schematics illustrating (upper panel) first and (lower panel) second types of transmission and deflection that appear in the ideal case, i.e., when the incident wave is completely converted into an outgoing deflected wave. R1 and R2 stand for the regular segments of the $\mathrm{PhC}$ that are adjacent to incidence and exit interfaces, respectively; IR stands for the irregular layer; oscillating and non-oscillating dependences indicate propagating-wave and evanescent-wave regimes.

not present in the resulting structure. The assumption of zero losses adopted here for the utilized dielectric materials allows using dimensionless variables. For the sake of convenience, we use the dimensionless variable $k L$, which, in fact, represents the normalized frequency. The material and geometrical parameters were chosen similar to some of our previous studies in order to demonstrate, among others, that the studied operation regimes may be achieved in very similar structures as some other well-known regimes.

\section{RESULTS AND DISCUSSION}

Figure 3 presents the general geometry of the studied structures, which are based on a $\mathrm{PhC}$ with dielectric rods. These structures only differ from each other in the location of the irregular layer.

\section{A. Structures with $\varepsilon=11.4$}

Transmittance results are shown for these structures in Fig. 4, in the case of a relatively small incidence angle, $\theta=14^{\circ}$. Permittivity of the rods, $\varepsilon=11.4$, is taken from the range to which many optical materials, such as GaAs and $\mathrm{Si}$, belong. A possible operation wavelength range can be chosen around $1.5 \mu \mathrm{m}$. In Fig. 4 and other figures that present the transmission results, the right and left arrows correspond to the upperside and lower-side illumination, respectively; the numbers 1

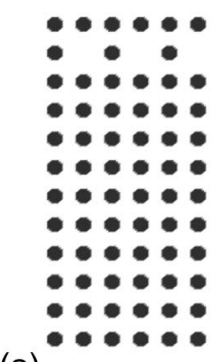

(a)
Fig. 3. Schematics of the structures with irregular layer that is (a) second, (b) third, and (c) fifth layer from the upper interface, within three periods of the irregular layer and the entire structure over the $x$-axis. 
and 2 indicate the deflection bands of the first and the second type (see the text below and Section 2), and the asterisks indicate the cases of higher-order transmission with efficiency higher than $90 \%$, in which strong single-beam deflection takes place. According to the general theory of diffraction gratings [8], the $m$ th order may propagate when

$$
|\sin \theta+2 \pi m /(k L)|<1 .
$$

In particular, it occurs at $k L>5.06$ for $m=-1$ when $\theta=14^{\circ}$. In turn, the orders $m=+1$ and $m=-2$ start to propagate at $k L=8.29$ and $k L=10.11$, respectively. In Fig. 4, one can see widebands, in which the order $m=-1$ dominates in transmission and, thus, a large portion of the incident wave energy contributes to single-beam deflection. Hence, blazing can be obtained without corrugations at the interfaces, as desired.

Two types of single-beam deflection bands can be distinguished here. For the first one, the existence of efficient deflection in the transmission mode is not affected by the location of the irregular layer. For example, it is observed in Figs. 4(a)-4(f) at $k L=7.1\left(\varphi=-40^{\circ}\right)$. Deflection bands of the second type are observed in Figs. 4(a) and 4(b) in the vicinity of $k L=6.6$ $\left(\varphi=-45.2^{\circ}\right)$. For these bands, strong dependence on the location of the irregular layer is typical. As follows from the obtained results (both shown and not shown), the difference between the first and the second types is related to whether the coupling of the incident wave to a wave propagating inside the $\mathrm{PhC}$ is possible or not, as explained in Section 2. Indeed, it can be possible for a given $\theta$ at the period $p=a$ for the former, whereas a larger period, i.e., $p=L=2 a$ is needed for the latter. In both cases, the irregular layer creates a propagating wave (due to the order $m=-1$ ) in the regular part of the $\mathrm{PhC}$, being adjacent to the exit half-space. The dependence of the band of the second type on the location of the irregular layer is connected, in fact, with the strength of damping (evanescence)
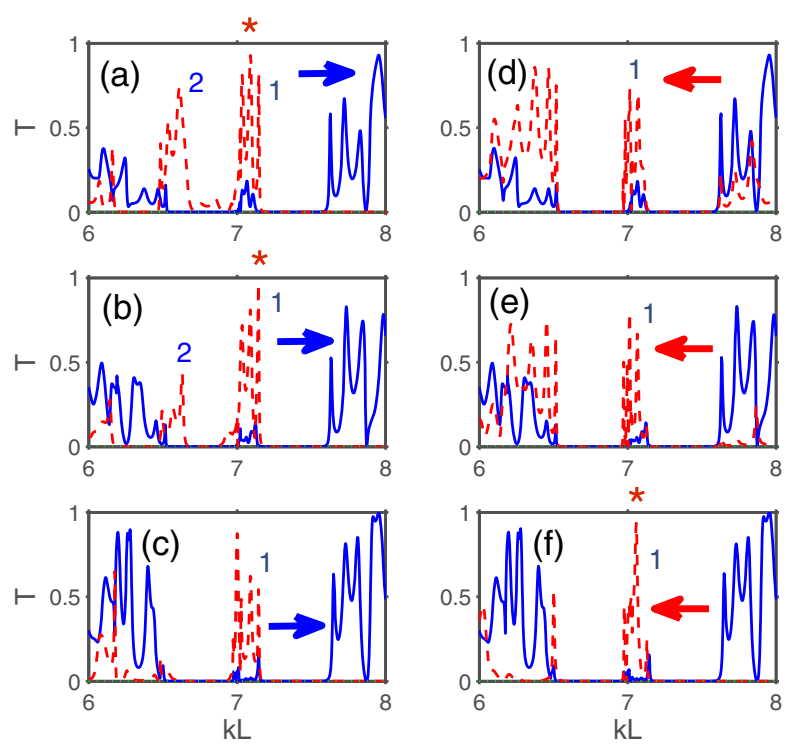

Fig. 4. Transmittance versus $k L$ for PhC-based structure with irregular layer ( $p=L=2 a$ ) that is (a), (d) second, (b), (e) third, and (c), (f) fifth layer from the upper interface at (a) $-(\mathrm{c})$ upper-side and (d)-(f) lower-side illumination. $d / a=0.4, \varepsilon=11.4$, and $\theta=14^{\circ}$. Solid blue line, $t_{0}$; dashed red line, (a) $-(\mathrm{c}) t_{-1}^{\rightarrow}$ and (d) $-(\mathrm{f}) t_{-1}^{\leftarrow}$. in the regular part of the $\mathrm{PhC}$, which is adjacent to the incidence interface. For this part, the propagating-wave regime requires $p>a$ [compare Figs. 4(a)-4(c)]. These differences affect the manifestations of deflection in the bands of the first and the second types.

The common effects of dispersion and diffraction enable the scenario when $T^{\rightarrow}=t_{-1}>0$ but $T^{\leftarrow}=t_{-1}^{\leftarrow}=0$, as occurs for the deflection bands of the second type. In contrast with the deflection bands of the first type, transmission vanishes for one of the two opposite incidence directions for bands of the second type. On the other hand, the former may allow us to obtain strong diffractions and related deflection, regardless of which side is illuminated and where the irregular layer is located.

For further evidence, Fig. 5 presents the examples of electric field distribution, which correspond to the cases of high transmittance and/or strong asymmetry in transmission that are shown in Fig. 4. In particular, cases (i) and (iii) correspond to the maxima of $t_{-1}$ observed within the deflection bands of the second type in Figs. 4(a) and 4(b), respectively. As expected, the field is damped in this case at the lower-side illumination, while it is not affected by the irregular layer [compare to Figs. 4(d) and 4(e)]. Cases (ii), (iv), and (v) correspond to the deflection bands of the first type. i.e., to the maximal values of $t_{-1}$ in Figs. 4(a) and 4(b) and to that of $t_{-1}^{\leftarrow}$ in Fig. 4(f), respectively, all in the vicinity of $k L=7$ (these cases are indicated by ${ }^{*}$ ).

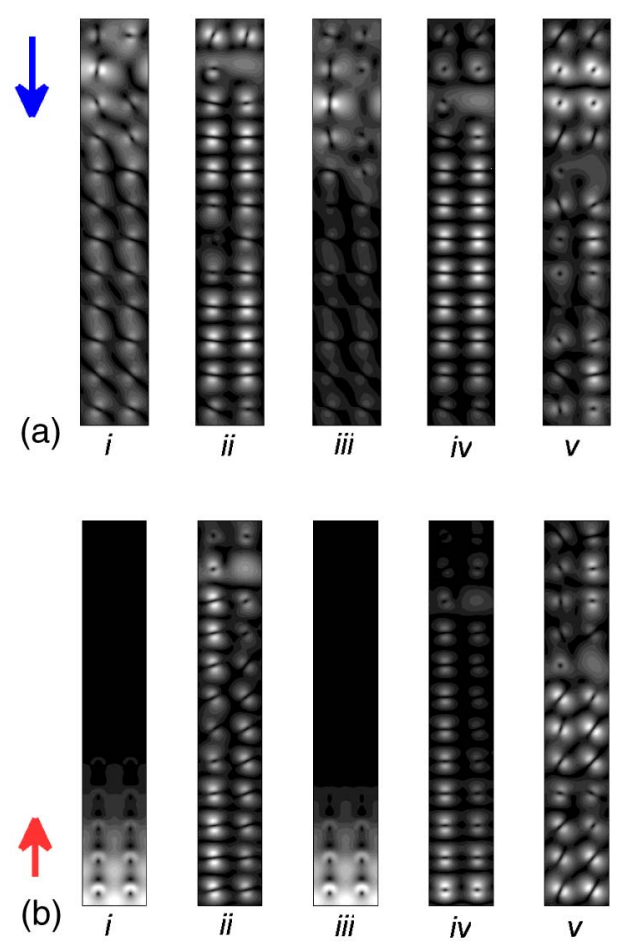

Fig. 5. Examples of electric field distribution within one period over $x, 0<x<L$, at $0<y<N a$, for $d / a=0.4, \varepsilon=11.4$, and $\theta=14^{\circ}$ at (a) upper-side illumination and (b) lower-side illumination. From the left to the right: (i) $k L=6.61$ and (ii) $k L=7.09$ when the second layer is removed, (iii) $k L=6.63$ and (iv) $k L=7.15$ when the third layer is removed, and (v) $k L=7$ when the fifth layer is removed. Centers of the rods are located at $x_{p}=a / 2+(p-1) a$ and $y_{s}=a / 2+(s-1) a, p=1,2$, and $1 \leq s \leq N$. The arrows schematically show which side of the structure is illuminated. 
One can see that the fields do not vanish over the entire thickness of the structure for both illumination directions, although they may be distinguished in strength of coupling and extent of contribution of different diffraction orders.

It is well known that the simplest way of tuning and switching deflections is realized by varying $\theta$ [38], which can strongly modify coupling and transmission scenarios but does not require changing the geometry of the structure or using an external control, like varying the voltage, temperature, or magnetostatic field. As an example, Fig. 6 presents the transmittance versus $k L$ for the same structure as in Fig. 4 but at $\theta=45^{\circ}$. Deflection bands of the second type appear now at $6.08<k L<6.49\left(-19^{\circ}<\varphi<-15.1^{\circ}\right)$. Nearly perfect deflection and asymmetric transmission are obtained near $k L=$ 6.23 when the irregular layer is the second one from the upper interface. This regime is indicated by an asterisk in Fig. 6(a). In this case, $T^{\rightarrow}=t_{-1}^{+}>0.97$ and $T^{\leftarrow}=t_{-1}^{\leftarrow}=t_{0}=0$, whereas $\varphi=-17.5^{\circ}$. Asymmetry in the transmission is strongly pronounced and deflection remains strong at a deeper embedding of the irregular layer, as occurs at $k L=6.23$ in Fig. 6(b).

The deflection bands of the first type are observed in Fig. 6 at $5.5<k L<6\left(-25.8^{\circ}<\varphi<-19.8^{\circ}\right)$. They typically represent sharp peaks that show low or moderate efficiency, e.g., $t_{-1}=0.44$ at $k L=5.87$ in Fig. $6(\mathrm{~b}) \quad\left(t_{0} \approx 0.02\right.$ and $\left.\varphi=-21.3^{\circ}\right), t_{-1}^{\leftarrow}=0.52$ at $k L=5.8$ in Fig. 6(d) $\left(t_{0} \approx 0.04, t_{-1} \approx 0\right.$, and $\left.\varphi=-22.1^{\circ}\right), t_{-1}^{\leftarrow}=0.4$ at $k L=$ 5.79 in Fig. $6(\mathrm{e})\left(t_{0} \approx 0, t_{-1}^{\rightarrow} \approx 0\right.$, and $\left.\varphi=-22.2^{\circ}\right)$, and $t_{-1}^{\leftarrow}=$ 0.47 at $k L=5.98$ in Fig. $6(\mathrm{e})\left(t_{0} \approx 0.04, t_{-1} \approx 0\right.$, and $\left.\varphi=-20.1^{\circ}\right)$. A very important and surprising feature is that the locations of the maxima of $t_{-1}$ at the upper-side illumination and those of $t_{-1}^{\leftarrow}$ at the lower-side illumination do not coincide in the general case. The most probable reason is that they depend, first of all, on the thickness of the regular segment of the $\mathrm{PhC}$ that is behind the embedded diffractive element and adjacent to the exit half-space. These deflection bands can also show well-pronounced asymmetry in transmission, whereas reflections are (nearly) perfect for one of the two opposite
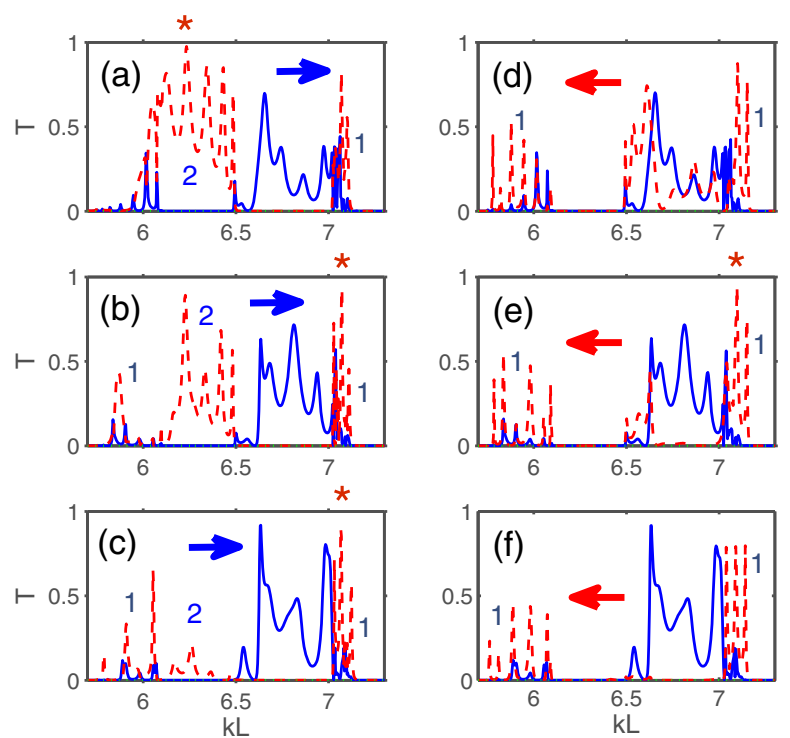

Fig. 6. Same as Fig. 4 but for $\theta=45^{\circ}$. incidence directions. However, high-efficiency deflection cannot be obtained because of the reflections.

At higher frequencies, e.g., at $k L=7.1$, the first-type transmission enables higher efficiency in the single-beam deflection regime. For example, for the rightmost peaks shown in Figs. 6(e) and 6(f), which are located at $k L=7.14, T^{\leftarrow}=$ $t_{-1}^{\leftarrow}=0.73\left(t_{-1}^{\rightarrow} \approx 0, T^{\rightarrow} \approx 0\right.$, and $\left.\varphi \approx-10^{\circ}\right)$ and $T^{\leftarrow}=$ $t_{-1}^{\leftarrow}=0.8\left(t_{0}=0, t_{-1} \approx 0\right)$, respectively. Hence, high-efficiency deflection and unidirectional transmission can coexist. These two cases are very interesting because the order $m=$ 0 that is expected to provide in-coupling is fully suppressed in the transmission. However, not all of the maxima of $t_{-1}$ and $t_{-1}^{\leftarrow}$, which are associated with the blazing and deflection observed in Fig. 6 at $k L>7$, correspond here to unidirectional transmission, i.e., transmission for the opposite incidence direction does not vanish. For example, $t_{-1}^{\leftarrow}=0.94$ at $k L=7.1$ in Fig. 6(e) but $t_{-1} \approx 0.15$ in Fig. 6(b) for the same $k L$. It is noteworthy that almost all incident wave energies can be converted into the -1 st-order deflected beam also at the upper-side illumination in the bands that do not show signatures of the second-type transmission. For instance, it is observed in Fig. $6(\mathrm{~b})$ at $k L=7.07$ where $t_{-1}>0.92$ (this regime is indicated by ${ }^{*}$ ), while $t_{-1}^{\leftarrow} \approx 0.25$ in Fig. 6(e) and $t_{0} \approx 0.06$. In fact, two cases could be distinguished beyond the second type, depending on whether the transmission tends to vanish for the opposite incidence direction or not.

Figure 7 presents the examples of electric field distribution for the cases with high transmittance and/or strong asymmetry in transmission from Fig. 6. Here, cases (ii) and (iii) correspond to the maxima of $t_{-1}$ that appear within the deflection bands of the second type in Figs. 6(a) and 6(b) (the first of them is indicated by ${ }^{*}$ ). The field vanishes and there is no effect of the irregular layer at the lower-side illumination. Cases (i), (iv), and (v) correspond to the deflection bands of the first type. The first case corresponds to the maximal value of $t_{-1}^{\leftarrow}$ in Fig. 6(d) at $k L<6$. The second and third cases correspond to the maxima of $t_{-1}$ in Fig. 6(b) at $k L>7$ and in Fig. 6(c) near $k L=6$, respectively (the first of them is indicated by ${ }^{*}$ ). Similarly to Fig. 5 , the fields are quite strong for the first type of transmission in both illumination directions. Thus, the strength of the coupling at the interfaces and the irregular layer can be critical for achieving efficient deflection and asymmetry in transmission.

A part of the above-discussed transmission features can be predicted by using the coupling analysis based on equifrequency dispersion contours (EFCs) in wave-vector space $[39,40]$. In turn, the existing transmission results can be better understood from the comparison with the dispersion results. Some examples are presented in Fig. 8. Coupling at the incidence interface takes place when the construction line for a given $k_{x}$ and, hence, for a given $k$ and $\theta$ crosses an $\mathrm{EFC}$ of the $\mathrm{PhC}$; if there is no crossing, in-coupling is impossible. Figures $8(\mathrm{a})-8(\mathrm{c})$ correspond to the case in Fig. 4 $\left(\theta=14^{\circ}\right)$, whereas Figs. 8(d)-8(f) correspond to the case in Fig. $6\left(\theta=45^{\circ}\right)$. The results shown in Figs. 8(a) and 8(b) predict the possibility of coupling to the order $m=-1$ for the period $p=L=2 a$, while there is no coupling for $p=a$ and no coupling for the order $m=0$ regardless of the period, which makes transmission and deflection of the second type 
(a)
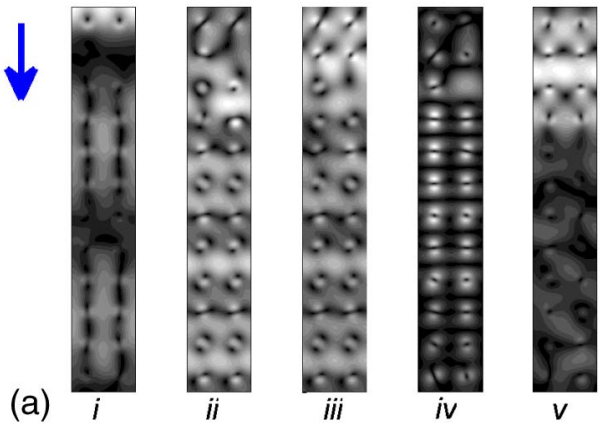

(b)
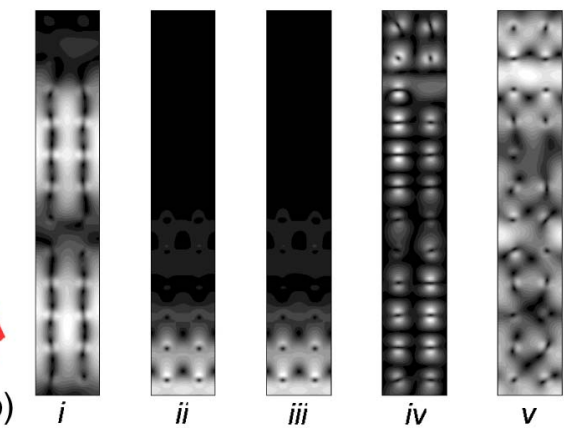

Fig. 7. Examples of electric field distribution within one period over $x, 0<x<L$, at $0<y<N a$, for $d / a=0.4, \varepsilon=11.4$, and $\theta=45^{\circ}$ at (a) upper-side illumination and (b) lower-side illumination. From the left to the right: (i) $k L=5.78$ and (ii) $k L=6.235$ when the second layer is removed, (iii) $k L=6.23$ and (iv) $k L=7.095$ when the third layer is removed, and (v) $k L=6.05$ when the fifth layer is removed; centers of the rods are located at $x_{p}=a / 2+(p-1) a$ and $y_{s}=a / 2+(s-1) a, p=1,2$, and $1 \leq s \leq N$. The arrows schematically show which side of the structure is illuminated.

possible. However, a comparison of Figs. 4(a)-4(c) with Figs. $8(\mathrm{a})$ and $8(\mathrm{~b})$ indicates that, in spite of the similarity of the coupling scenarios predicted by EFCs, there is a strong difference in the transmissions between the ranges near $k L=$ $6.6\left[\max T \rightarrow \approx 0.73\right.$ and $\max T^{\rightarrow} \approx 0.43$ in Figs. 4(a) and 4(b)] and near $k L=6.8\left[\max T^{\rightarrow} \approx 0.08\right.$ and $\max T^{\rightarrow}<$ 0.01 in Figs. 4(a) and 4(b)], where it is very strong. According to the predictions based on the EFC results in Fig. 8(c), both orders $m=0$ (at $p=a$ and $p=2 a$ ) and $m=$ -1 (at $p=2 a$ ) may contribute to the transmission. Indeed, this situation is observed in Figs. 4(a)-4(f) at $k L=7.05$, where the first-type transmission and deflection are realized. Figure 8(d) illustrates the dispersion behavior typical for $5.7<k L<6$, i.e., when both orders $m=0$ (at $p=a$ and $p=2 a$ ) and $m=-1$ (at $p=2 a$ ) may be coupled at $\theta=45^{\circ}$. Hence, the order and direction selectivity observed in this $k L$ range in Fig. 6 strongly depend on effects that are not connected with the dispersion. Figure $8(\mathrm{e})$ predicts that the order $m=-1$ may be coupled at $p=2 a$, while the order $m=0$ is always uncoupled at $k L=6.2$. Finally, Fig. 8(f) illustrates one more case in which the orders $m=0$ and $m=-1$ are allowed to be coupled at $p=$ $a$ and $p=2 a$, and at $p=2 a$, respectively. From the comparison with Fig. 6, it can be expected that suppression of the order $m=0$ and contribution of the order $m=-1$ to $T^{\leftarrow}$ at $k L=$ 7.05 and $\theta=45^{\circ}$ will appear, owing to diffraction effects.

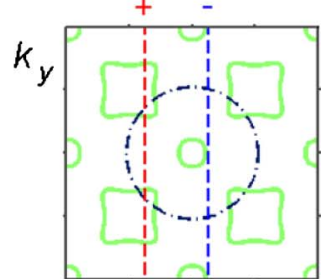

(a)

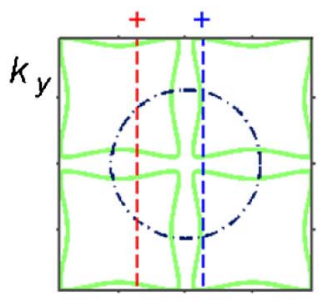

(c)

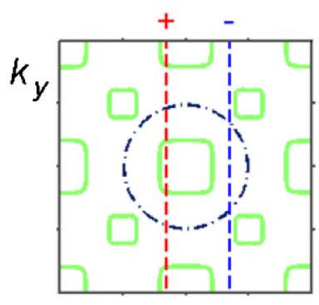

(e)

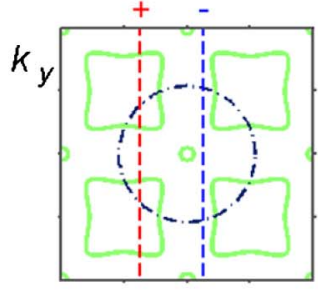

(b)
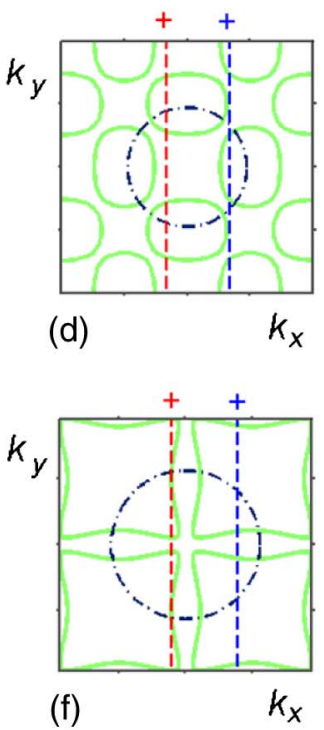

Fig. 8. EFCs in $\left(k_{x}, k_{y}\right)$ plane for the regular infinite $\mathrm{PhC}$ with $d / a=0.4$ and $\varepsilon=11.4$ (solid lines) and air (dashed-dotted lines) at (a) $k L=6.6$, (b) $k L=6.8$, and (c) $k L=7.05$ for $\theta=14^{\circ}$, and (d) $k L=5.98$, (e) $k L=6.2$, and (f) $k L=7.05$ for $\theta=45^{\circ}$. Signs + and - indicate the cases when coupling is possible or impossible, respectively, for $m=0$ and for $m=-1$ at $p=L=2 a$; impossibility of coupling for the order $m=-1$ at $p=a$ is provided by parameter adjustment. Construction lines (vertical dashed lines) correspond to the orders $m=0$ (right blue lines) and $m=-1$ at $p=L=2 a$ (left red lines), respectively; $k_{x}$ and $k_{y}$ vary from $-2 \pi / a$ to $2 \pi / a$ in all plots.

Generally, the transmission results remain more indicative because the coupling strength cannot be predicted by the EFC analysis, at least in its conventional and easily applicable form. Moreover, zero- $n$ and negative near-zero- $n$ regimes are among those of interest but are not properly predictable by the EFC analysis.

\section{B. Structures with $\varepsilon=9.61$}

To illustrate the possibility of obtaining similar operation regimes when using materials that might be more applicable at terahertz frequencies, Fig. 9 presents the results in the case when the rods are made of a material with $\varepsilon=9.61\left(\mathrm{Al}_{2} \mathrm{O}_{3}\right.$ below $2 \mathrm{THz}$ ). The values of $d / a$ and $\theta$ are a bit larger than in Fig. 6 in order to obtain more illustrative results. Although the basic features are the same as in Fig. 6, there are important differences, which include stronger transmission in the second-type deflection band at the deep embedding of the irregular layer. For instance, at $k L=6.05$, we obtain $T^{\rightarrow}=$ $t_{-1}=0.38$ in Fig. 9(c), i.e., when the thickness of the 

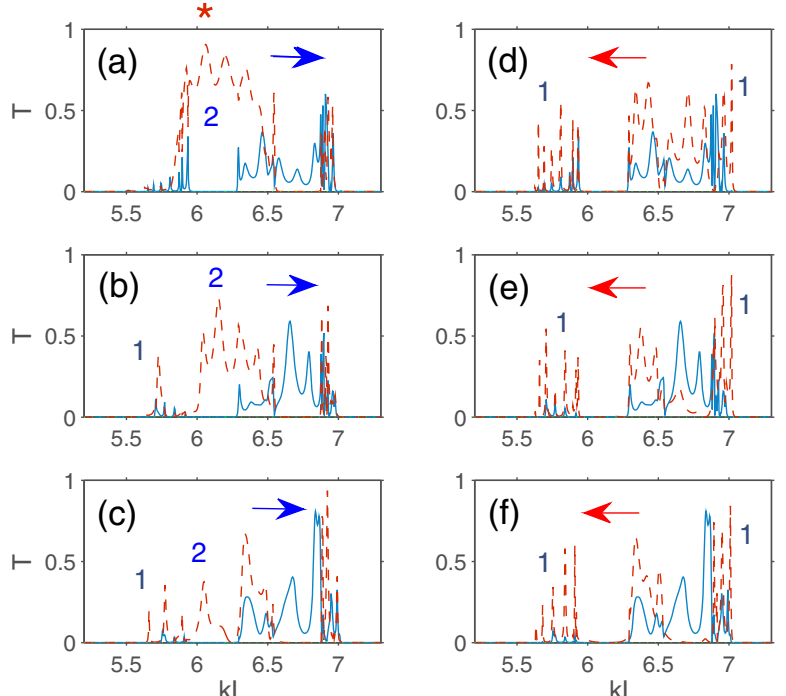

Fig. 9. Transmittance versus $k L$ for structure with irregular layer ( $p=L=2 a$ ) that is (a), (d) second, (b), (e) third, and (c), (f) fifth layer from the upper interface at (a)-(c) upper-side and (d)-(f) lowerside illumination. $d / a=0.45, \varepsilon=9.61$, and $\theta=47^{\circ}$. Solid blue line, $t_{0}$; dashed red line, (a)-(c) $t_{-1}$ and (d) $-(\mathrm{f}) t_{-1}^{\leftarrow}$.

evanescent-wave segment is rather large, while $T^{\leftarrow}=0$ in Fig. 9(d). Wideband single-beam deflection and asymmetric transmission with $T^{\rightarrow}=t_{-1}>0.62$ and $T^{\leftarrow} \approx 0$ are observed at $5.94<k L<6.28\left(-19^{\circ}<\varphi<-15.6^{\circ}\right)$ when the irregular layer is the second layer from the upper interface [see Figs. 9(a) and $9(\mathrm{~d})]$. In Fig. 9(a), $\max t_{-1}=0.9$ at $k L=6.06$ $\left(\theta=-17.8^{\circ}\right)$ is indicated by an asterisk.

For the first-type deflection bands located in Fig. 9 at $5.6<$ $k L<6$ and near $k L=7$, the transmission behavior is also similar to that observed for such bands in Fig. 6. In particular, the similarity is related to the difference in the locations of the
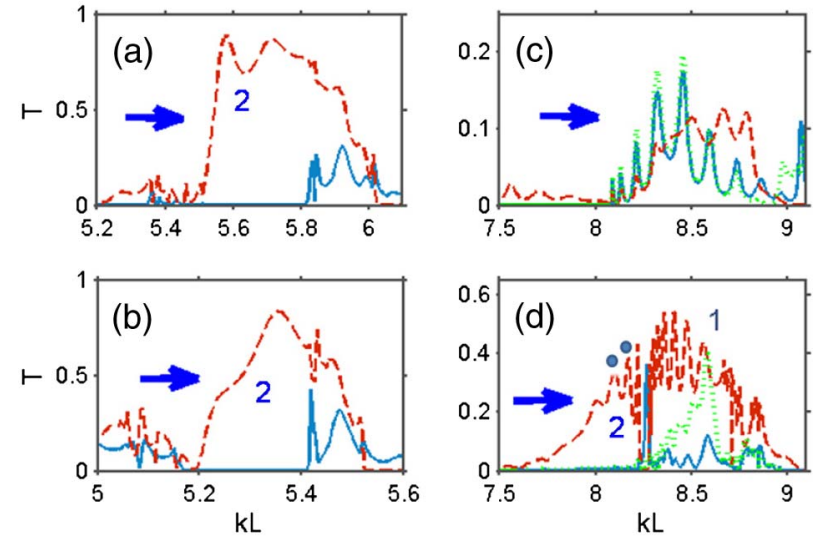

Fig. 10. Transmittance within two selected $k L$-ranges for two structures with irregular layers $(p=L=2 a)$ that is the second layer from the upper interface at the upper-side illumination; (a), (c) $d / a=0.5$, (b), (d) $d / a=0.55, \varepsilon=9.61$, and $\theta=47^{\circ}$. Solid blue line, $t_{0}$; dashed red line, $t_{-1}$; and (c), (d) green dotted line, $t_{-2}$. Note that $t_{0} \approx$ $\overrightarrow{t_{-2}}$ in plot (c) at $8<k L<8.8$. maxima of $t_{-1}$ and $t_{-1}^{\leftarrow}$ that can lead to strong asymmetry in the transmission, even though the order $m=0$ may be incoupled. For example, $t_{-1}^{\leftarrow}=0.57$ at the peak located at $k L=$ 5.84 in Fig. 9(f), whereas $t_{0} \approx 0.04$ and $t_{-1} \approx 0.02$ in Fig. 9(c) at the same $k L$, and $t_{-1}^{\leftarrow}=0.61$ at the peak located at $k L=$ 5.91 in Fig. 9(f), whereas $t_{0}=0.036$ and $t_{-1}=0.025$ in Fig. 9 (c) at the same $k L$. One should also notice the peaks of $t_{-1}^{\leftarrow}$ at $5.6<k L<5.9$ in Fig. 9(d), including the peak of $t_{-1}^{\leftarrow} \approx 0.45$ at $k L \approx 5.65\left(t_{0} \approx 0\right)$, and the weak maxima of $t_{-1}$ and $t_{0}$ at $k L<5.8$ in Fig. 9(a).

Next, we demonstrate deflection bands of the second type that enable (i) dual-band single-beam deflection, and (ii) highefficiency single-beam deflection at the deep embedding of the irregular layer (this is possible for the rod-type $\mathrm{PhC}$ with $\varepsilon=9.61$ ). In Fig. 10, the results are presented for three structures, with the irregular layer being the second one from the upper interface, which differ from those in Fig. 9 in the value of $d / a$. The structure with $d / a=0.5$ allows one to obtain high-efficiency wideband single-beam deflection, but finding a proper second band at higher frequencies remains problematic [see Figs. 10(a) and 10(c)]. Thus, the structures with $d / a=0.55$ are more suitable [see Figs. $10(\mathrm{~b})$ and $10(\mathrm{~d})$ ]. For example, $T^{\rightarrow}=t_{-1}>0.62$ when $5.31<k L<5.41$ $\left(-26.9^{\circ}<\varphi<-25.5^{\circ}\right)$ in Fig. $10(\mathrm{~b}) ; T^{\rightarrow} \approx t_{-1}=0.35$ and 0.39 for the maxima observed in Fig. $10(\mathrm{~d})$ at $k L=8.1$ and 8.15 , respectively (both indicated by circles). Additional optimization is required for obtaining max $t_{-1}>0.6$ for both bands simultaneously. Note that the asymmetry in the transmission is quite strongly pronounced for the deflection bands in Figs. 10 (a) and 10 (b), i.e., $T \leftarrow \approx 0$.

Now, let us discuss the possibility of obtaining highefficiency single-beam deflection at the deep embedding of the irregular layer in bands of the second type. Above, it has been shown for first-type deflection bands. In Fig. 11, it is demonstrated for second-type deflection bands. Such widebands are located at $5.52<k L<5.75\left(-24^{\circ}<\varphi<-21.2^{\circ}\right)$ with $\max t_{-1}=0.53$ in Fig. 11 (a) and at $5.2<k L<5.4$ $\left(-28.5^{\circ}<\varphi<-25.6^{\circ}\right)$ with $\max t_{-1}=0.45$ in Fig. 11(b).
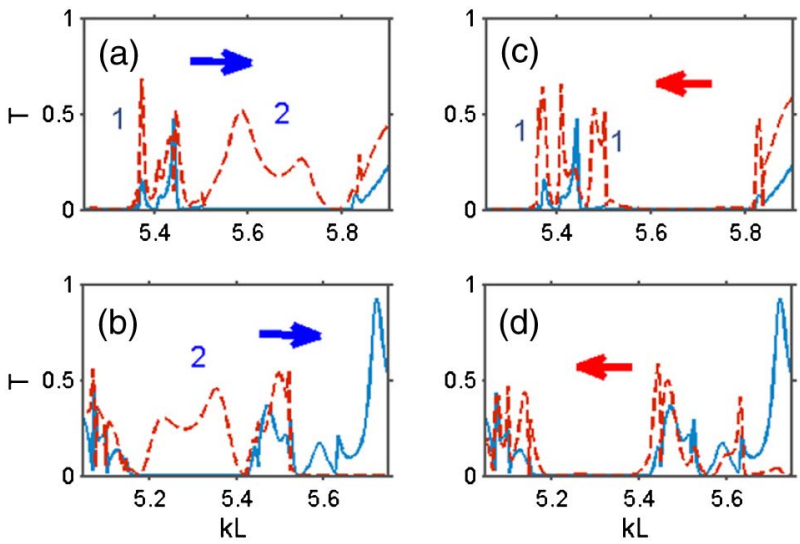

Fig. 11. Transmittance versus $k L$ for structures with irregular layers $(p=L=2 a)$ that is the fourth layer from the upper interface, which are illuminated from (a, b) upper side and (c, d) lower side. (a), (c) $d / a=0.5$, (b), (d) $d / a=0.55, \varepsilon=9.61$, and $\theta=47^{\circ}$. Solid blue line, $t_{0}$; dashed red line, (a), (b) $t_{-1}^{\rightarrow}$ and (c), (d) $t_{-1}^{\leftarrow}$. 
(a)
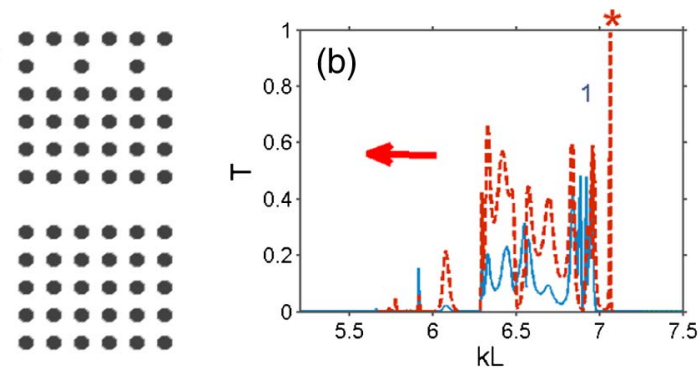

Fig. 12. (a) Schematic of the structure with the irregular layer $(p=L=2 a)$ that is the second layer and an additional line defect that is the seventh layer from the upper interface. (b) Transmittance versus $k L$ at lower-side illumination. $d / a=$ $0.45, \varepsilon=9.61$, and $\theta=47^{\circ}$. Solid blue line, $t_{0}$; dashed red line, $t_{-1}^{\leftarrow}$.

A similar band can be obtained for $d / a=0.6$ at $4.95<k L<$ $5.1\left(-32.5^{\circ}<\varphi<-30^{\circ}\right)$ with $\max t_{-1}=0.58$. Hence, wideband deflection and unidirectional transmission can be obtained for the second-type bands also at the deep embedding. In this case, weak damping in the regular part of the PhC, which is located between the incidence interface and the irregular layer, is critical. It is noteworthy that the advancements presented in Figs. 10 and 11 have not yet been found in the structures with $\varepsilon=11.4$ but are expected to exist at different values of $d / a$.

Finally, we consider the possibility of single-beam deflection and asymmetric transmission in structures with a more complex irregular part. Now, we introduce an additional line defect by removing one layer of the rods in order to separate two groups of the rod layers that belong to the thicker regular part of the PhC. Figure 12 presents the transmittance versus $k L$ for the structure, which differs from those in Figs. 9(a) and 9(d) in that a line defect is added. A wide second-type deflection band appears for this structure at the upper-side illumination at $5.92<k L<6.29\left(-19.3^{\circ}<\varphi<-15.5^{\circ}\right)$, where $T^{\rightarrow} \approx t_{-1}$ and $\max t_{-1}=0.91$ at $k L=6.12\left(\varphi=-17.2^{\circ}\right)$, but a large part of it is not a unidirectional transmission band. Due to the line defect, a low-efficiency deflection band with $T^{\leftarrow}>$ 0 is observed near $k L=6.07$ in Fig. 12(b). A similar band with $T^{\rightarrow}>0$ appears near $k L=7.38$ at the upper-side illumination (not shown).

A very interesting regime is observed in Fig. 12(b), for which $T^{\leftarrow}=t_{-1}^{\leftarrow}>0.99$ and $T^{\rightarrow} \approx 0$ at $k L=7.06$ that yields $\varphi=-9.1^{\circ}$; it is indicated by an asterisk. Thus, it is possible to obtain nearly perfect single-beam deflection and nearly perfect asymmetry in transmission, even if the structure is nonsymmetric and does not have corrugations at the interfaces. A detailed theoretical study is required to entirely understand the underlying physics of this regime. Similar features have been observed for the structures in Figs. 4 and 6, which are modified by adding a line defect. However, such a high transmittance as at $k L=7.06$ in Fig. 12(b) has not been found. The use of additional line defects promises more freedom for possible combinations of different operating regimes that are realized in different frequency ranges in one structure. It is worth noting that the structures with two and more defect layers are appropriate for widening a high-efficiency deflection band.
The main mechanism of widening is expected to be based, in this case, on merging narrow transmission bands. Its study is beyond the scope of this paper.

\section{CONCLUDING REMARKS}

To summarize, we have studied transmission-mode singlebeam deflection in nonsymmetric finite-thickness structures without corrugations at the interfaces, which are based on rod-type dielectric $\mathrm{PhCs}$ and include an embedded irregular but still periodic rod layer. The principal possibility of the efficient conversion of the incident wave energy to the first negative order, i.e., efficient blazing and relevant single-beam deflection, have been demonstrated. Two types of transmission and deflection have been compared. Deflection of the first type, which is connected with zero-order coupling at the interfaces and the consequent redistribution of the wave energy between diffraction orders at the irregular layer, can remain strong whatever the depth of embedding is. This feature can be especially important when the range of variation of geometrical and material parameters is limited due to the requirements related to other simultaneous functionalities. The second type is associated with the unidirectional regime of asymmetric transmission arising when the zero order may not be coupled at the structure period that is equal to the lattice constant and, thus, a higher order may propagate only due to the transformation of the evanescent wave at the irregular layer. Although the achievable efficiency is usually higher in the case when the irregular layer is located closer to one of the interfaces, it may remain quite high also when this layer is deeply embedded. Moreover, the deflection bands of the two types can coexist in one structure, including the case when they appear in neighboring frequency ranges. Generally speaking, the main operating regimes are similar in many senses to those studied earlier in nonsymmetric $\mathrm{PhC}$ gratings that have corrugations at the interfaces. In addition, new regimes were found that differ from those presently known for (but are expected to be obtainable in) $\mathrm{PhC}$ gratings. A deeper study of the basic physics is required in order to detect whether and how widebands of high-efficiency single-beam deflection could be obtained in the proposed structures when using the first-type transmission and then properly combined with the deflection bands related to the second-type transmission. The obtained results indicate a route to lowprofile multifunctional photonic devices in which single-beam deflection and other functionalities can be realized within different frequency ranges. The suggested approach is expected to be usable for PhC slab technology. Understanding physical conditions of perfect deflection and implementing this approach in the framework of multilayer technology will be the subjects of our future studies.

Funding. Narodowe Centrum Nauki (NCN) (National Science Center of Poland) (MetaSel DEC-2015/17/B/ST3/ 00118); Türkiye Bilimsel ve Teknolojik Araştirma Kurumu (TÜBİTAK) (107A004, 109A015， 109E301); European Science Foundation (ESF) (EPIGRAT); Devlet Planlama Örgütü (DPT) (HAMIT); North Atlantic Treaty Organization (SET-181); Department of Education, Australian Government. 
Acknowledgment. The contribution of A. E. S. was partially supported by the Australian Government under the Endeavour Fellowship Programme. E. O. acknowledges partial support from the Turkish Academy of Sciences.

\section{REFERENCES}

1. D. Maystre, "Photonic crystal diffraction gratings," Opt. Express 8, 209-216 (2001).

2. A. E. Serebryannikov and E. Ozbay, "One-way Rayleigh-Wood anomalies and tunable narrowband transmission in photonic crystal gratings with broken structural symmetry," Phys. Rev. A 87, 053804 (2013).

3. A. Mandatori, M. Bertolotti, and C. Sibilia, "Asymmetric transmission of some two-dimensional photonic crystals," J. Opt. Soc. Am. B 24, 685690 (2007)

4. A. E. Serebryannikov, A. O. Cakmak, and E. Ozbay, "Multichannel optical diode with unidirectional diffraction relevant total transmission," Opt. Express 20, 14980-14990 (2012).

5. L. Zinkiewicz, J. Haberko, and P. Wasylczyk, "Highly asymmetric near infrared light transmission in an all-dielectric grating-on-mirror photonic structure," Opt. Express 23, 4206-4211 (2015).

6. S. Collardey, A.-C. Tarot, P. Pouliguen, and K. Mahdjoubi, "Use of electromagnetic band-gap materials for RCS reduction," Microwave Opt. Technol. Lett. 44, 546-550 (2005).

7. A. E. Serebryannikov, T. Magath, and K. Schuenemann, "Bragg transmittance of s-polarized waves through finite-thickness photonic crystals with periodically corrugated interface," Phys. Rev. E 74 066607 (2006).

8. R. Petit, Electromagnetic Theory of Gratings (Springer, 1980).

9. A. Figotin and I. Vitebskiy, "Electromagnetic unidirectionality in magnetic photonic crystals," Phys. Rev. B 67, 165210 (2003).

10. F. D. M. Haldane and S. Raghu, "Possible realization of directional optical waveguides in photonic crystals with broken time-reversal symmetry," Phys. Rev. Lett. 100, 013904 (2008).

11. A. E. Serebryannikov and E. Ozbay, "Unidirectional transmission in non-symmetric gratings containing metallic layers," Opt. Express 17, 13335-13345 (2009).

12. A. E. Serebryannikov, E. Ozbay, and S. Nojima, "Asymmetric transmission of terahertz waves using polar dielectrics," Opt. Express 22, 3075-3088 (2014).

13. Y. Fu, L. Xu, Z. H. Hang, and H. Chen, "Unidirectional transmission using array of zero-refractive-index metamaterials," Appl. Phys. Lett. 104, 193509 (2014)

14. P. Rodriguez-Ulibarri, M. Beruete, M. Navarro-Cia, and A. E. Serebryannikov, "Wideband unidirectional transmission with tunable sign-switchable refraction and deflection in nonsymmetric structures," Phys. Rev. B 88, 165137 (2013).

15. M. J. Lockyear, A. P. Hibbins, K. R. White, and J. R. Sambles, "Oneway diffraction grating," Phys. Rev. E 74, 056611 (2006).

16. C. Wang, C.-Z. Zhou, and Z.-Y. Li, "On-chip optical diode based on silicon photonic crystal heterojunctions," Opt. Express 19, 2694826955 (2011).

17. J. H. Oh, H. W. Kim, P. S. Ma, H. M. Seung, and Y. Y. Kim, "Inverted bi-prism phononic crystals for one-sided elastic wave transmission applications," Appl. Phys. Lett. 100, 213503 (2012).

18. J. H. Shi, H. F. Ma, C. Y. Guan, Z. P. Wang, and T. J. Cui, "Broadband chirality and asymmetric transmission in ultrathin $90^{\circ}$-twisted Babinetinverted metasurfaces," Phys. Rev. B 89, 165128 (2014).

19. C. Pfeiffer, C. Zhang, V. Ray, L. J. Guo, and A. Grbic, "High performance bianisotropic metasurfaces: asymmetric transmission of light," Phys. Rev. Lett. 113, 023902 (2014).
20. S. Cakmakyapan, A. E. Serebryannikov, H. Caglayan, and E. Ozbay, "Spoof-plasmon relevant one-way collimation and multiplexing at beaming from a slit in metallic grating," Opt. Express 20, 2663626648 (2012).

21. E. Battal, T. A. Yogurt, and A. K. Okyay, "Ultrahigh contrast one-way optical transmission through a subwavelength slit," Plasmonics 8, 509-513 (2013).

22. F. V. Liu, D. A. B. Miller, and S. Fan, "Ultra-compact photonic crystal waveguide spatial mode converter and its connection to the optical diode effect," Opt. Express 20, 28388-28397 (2012).

23. H. Ye, D. Wang, Z. Yu, J. Zhang, and Z. Chen, "Ultra-compact broadband mode converter and optical diode based on linear rod-type photonic crystal waveguide," Opt. Express 23, 9673-9680 (2015).

24. H. Kurt, D. Yilmaz, A. E. Akosman, and E. Ozbay, "Asymmetric light propagation in chirped photonic crystal waveguides," Opt. Express 20, 20635-20646 (2012).

25. L. Feng, M. Ayache, J. Huang, Y.-L. Xu, M.-H. Lu, Y. F. Chen, Y. Fainman, and A. Scherer, "Nonreciprocal light propagation in a silicon photonic circuit," Science 333, 729-733 (2011).

26. Y.-L. Xu, L. Feng, M.-H. Lu, and Y.-F. Chen, "Asymmetric optical mode conversion and transmission by breaking PT-symmetry on silicon photonic circuits," Phys. Lett. A 376, 886-890 (2012).

27. M. C. Hutley, Diffraction Gratings (Academic, 1982).

28. P. Lalanne, S. Astilean, P. Chavel, E. Cambril, and H. Launois, "Design and fabrication of blazed binary diffractive elements with sampling periods smaller than the structural cutoff," J. Opt. Soc. Am A 16, 1143-1156 (1999).

29. P. Lalanne, "Waveguiding in blazed-binary diffractive elements," J. Opt. Soc. Am A 16, 2517-2520 (1999).

30. C. Ribot, M.-S. L. Lee, S. Collin, S. Bansropun, P. Plouhinec, D. Thenot, S. Cassette, B. Loiseaux, and P. Lalanne, "Broadband and efficient diffraction," Adv. Opt. Mater. 1, 489-493 (2013).

31. C. Pfeiffer and A. Grbic, "Metamaterial Huygens' surfaces: tailoring wave fronts with reflectionless sheets," Phys. Rev. Lett. 110 197401 (2013).

32. A. Epstein and G. Eleftheriades, "Huygens' metasurfaces via the equivalence principle: design and applications," J. Opt. Soc. Am. B 33, A31-A50 (2016).

33. E. Colak, A. E. Serebryannikov, P. V. Usik, and E. Ozbay, "Diffraction inspired unidirectional and bidirectional beam splitting in defectcontaining photonic structures without interface corrugations," J. Appl. Phys. 119, 193108 (2016).

34. K. Inoue and K. Othaka, eds., Photonic Crystals: Physics, Fabrication and Applications (Springer, 2004).

35. T. Magath and A. E. Serebryannikov, "Fast iterative, coupledintegral-equation technique for inhomogeneous profiled and periodic slabs," J. Opt. Soc. Am. A 22, 2405-2418 (2005).

36. See www.cst.com for software details.

37. W.-M. Ye, X.-D. Yuan, C.-C. Guo, and C. Zen, "Unidirectional transmission in non-symmetric gratings made of isotropic material," Opt. Express 18, 7590-7595 (2010).

38. J. H. Wu, L. K. Ang, A. Q. Liu, H. G. Teo, and C. Lu, "Tunable high-Q photonic-bandgap Fabry-Perot resonator," J. Opt. Soc. Am. B 22, 1770-1777 (2005).

39. A. E. Serebryannikov, E. Colak, A. Petrov, P. V. Usik, and E. Ozbay, "Multifrequency spatial filtering: a general property of two-dimensional photonic crystals," Photon. Nanostr. Fundam. Appl. 18, 1-9 (2016).

40. S. Foteinopoulou and C. M. Soukoulis, "Electromagnetic wave propagation in two-dimensional photonic crystals: a study of anomalous refractive effects," Phys. Rev. B 72, 165112 (2005). 